\title{
A REMARK ON CONTINUITY CONDITIONS ${ }^{1}$
}

\section{P. B. KENNEDY}

In a recent paper $[1$, p. 207], as a corollary to a theorem on Fourier series with gaps, I pointed out that a function of one real variable may satisfy a Lipschitz condition in a set of positive measure without satisfying such a condition in any interval. Here a Lipschitz condition in a general set of real numbers is defined as follows: $f(x) \in \operatorname{Lip} \alpha$ in $E$ if

$$
f(x+h)-f(x)=O\left(|h|^{\alpha}\right)
$$

uniformly for $x$ in $E$, as $h \rightarrow 0$ through unrestricted real values. When writing [1] I overlooked the fact that it is possible by simpler methods to obtain a much stronger result. Let $E$ be a subset of $(0,1), f(x)$ a function defined in $(0,1)$ and $\omega(t)$ a function defined, positive and monotonic increasing in $(0,1)$ and satisfying $\omega(t) \rightarrow 0$ as $t \rightarrow 0$. Let us then call $\omega(t)$ a modulus of continuity of $f(x)$ in $E$ if

$$
|f(x+h)-f(x)| \leqq \omega(|h|)
$$

for all $x$ in $E$ and all $x+h$ in $(0,1)(h \neq 0)$. With this terminology we have the following theorem.

Theorem. Let $E$ be any subset whatever of $(0,1)$. Let $\omega(t)$ be positive and monotonic increasing in $(0,1)$ and satisfy $\omega(t) \rightarrow 0$ as $t \rightarrow 0$. Then there exists a function $f(x)$ defined in $(0,1)$ such that

(i) $\omega(t)$ is a modulus of continuity of $f(x)$ in $E$, E.

(ii) $f(x)$ is discontinuous at every interior point of the complement of

Here and in the rest of this note, "complement" means "complement with respect to $(0,1)$." In the theorem we may, for instance, choose for $E$ a nondense closed set of positive measure, whose complement is then an open set dense in $(0,1)$. Thus we see from the theorem that $f(x)$ may satisfy as strong a continuity condition as we wish in $E$, without even being continuous in any interval.

To prove the theorem, denote by $S$ the interior of the complement of $E$. We assume that $S$ is nonempty, since if this is not the case the function $f(x) \equiv 0$ has all the required properties. Then $S$ is the union of countably many disjoint open intervals, say

Received by the editors August 7, 1958.

1 Research sponsored by the Air Research and Development Command, United States Air Force, through its European Office, under Contract No. AF 61 (514) - 1399. 


$$
S=\bigcup_{n=1}^{\infty}\left(x_{n}-\delta_{n}, x_{n}+\delta_{n}\right) .
$$

Let $T$ denote the complement of $S$, so that $E \subset T$. Put $f(x)=0$ in $T$, and also put $f(x)=0$ when $x$ is a rational point of $S$. If $x$ is an irrational point of $S$, then $\left|x-x_{n}\right|<\delta_{n}$ for exactly one value of $n$; in this case put

$$
f(x)=\omega\left(\delta_{n}-\left|x-x_{n}\right|\right) .
$$

Then $f(x)$ is defined throughout $(0,1)$; we now prove (i) and (ii).

Suppose that $x \in E$ and $x+h \in(0,1)(h \neq 0)$. If either $x+h \in T$ or $x+h$ is a rational point of $S$, then (1) is trivial. Otherwise $x+h$ is an irrational point of $S$, and so of some interval $\left(x_{n}-\delta_{n}, x_{n}+\delta_{n}\right)$, and

$$
f(x+h)-f(x)=\omega\left(\delta_{n}-\left|x+h-x_{n}\right|\right) .
$$

But obviously $|h|$ is not less than the distance of $x+h$ from the nearer of the two points $x_{n}-\delta_{n}, x_{n}+\delta_{n}$; and this distance is $\delta_{n}$ $-\left|x+h-x_{n}\right|$. Hence (1) is true, by (2) and the monotonicity of $\omega(t)$. This proves (i).

(ii) is plainly true, from the definitions of $\omega(t)$ and $f(x)$. This proves the theorem.

I wish to thank Professor W. W. Rogosinski for calling my attention to an error in an earlier version of this note.

\section{REFERENCE}

1. P. B. Kennedy, On the coefficients in certain Fourier series, J. London Math. Soc. vol. 33 (1958) pp. 196-207.

University College, Cork, Ireland 